gain-of-function model, TGM2 was upregulated in HMLE-E2 cells and compared to shTGM2 knockdown BM cells. Growth rates were analyzed using metabolic activity over 8 days, and drug sensitivity to Neratinib (0-1000 nM) was analyzed via cell titer. To account for the different transport properties of the 3 distinct culture environments, we developed a mathematical model for each condition, allowing us to normalize the drug sensitivity results across models to effectively compare true biological resistivity. RESULTS/ANTICIPATED RESULTS: We observed that increased cellular levels of TGM2 significantly increase the growth rate and drug resistivity of cells on fibronectin matrices. Interestingly, in 2D cultures, TGM2 expression was correlated with higher Neratinib resistivity but did not affect growth rates. In spheroid models without a significant matrix component, that rely solely on cell-cell junctions, high levels of TGM2 were correlated with lower survival rates. Lower levels of TGM2 are correlated with a more epithelial phenotype, and using our mathematical model we have identified significant transport differences between high and low TGM2 spheroids. We theorize that the low TGM2 spheroids have denser packing, which lowers the rate of diffusion and, thus reduces the effective concentration of the drug to the majority of the cells. DISCUSSION/SIGNIFICANCE OF IMPACT: Our studies indicate that the cellular response to drugs can be altered by changes in both transport properties of the tissue and the CM interactions. By systematically investigating the effects of CC interactions and CM interactions, we can use mathematical models to delineate physical means of drug resistivity from a biologically driven resistance.

\section{Regulatory Science}

\section{Implementation of Real-World Data and Real-World} Evidence in Clinical Studies

Jessica Pham ${ }^{1}$, and Eunjoo Pacifici ${ }^{1}$

${ }^{1}$ University of Southern California

OBJECTIVES/GOALS: Real-world studies have been gaining momentum in providing evidence of treatment effectiveness and hold great potential for facilitating the drug regulatory process. The U.S. Food and Drug Administration (FDA) has recognized this by providing a framework for using real-world data (RWD) to generate real-world evidence (RWE). The objective of this study is to assess the current level of RWE implementation in clinical studies. METHODS/STUDY POPULATION: Using keywords relevant to RWE, we reviewed studies on drugs, biologics, and medical devices published on PubMed in 2018. Information regarding the therapeutic area of focus, intervention type, study design, primary outcome, and data source was recorded. Further analyses of the three main therapeutic areas of study (oncology, cardiology, and infectious diseases) were performed to determine how RWE was being utilized. In addition, a broad "real-world" search was performed on Clinicaltrials.gov, from which we extracted relevant observational and Phase I, II, II/III, III, III/IV, and IV studies. A supplemental PubMed search was used to evaluate published studies in order to identify which field these trials were concentrated in and the outcome of interest.” RESULTS/ANTICIPATED RESULTS: After application of "real-world" search terms to PubMed, 995 hits were generated and of these, 311 studies were excluded. More than half of the studies were observational and retrospective in nature (64\%) with $70 \%$ examining drug/biologic outcomes. RWE data sources were largely dominated by medical records and claims data. The primary uses of RWE across oncology, cardiology, and infectious diseases included supporting drug product effectiveness, assessing safety, and evaluating treatment patterns. Of the 207 RWE studies identified on ClinicalTrials.gov, 66 were cancer randomized controlled trials (RCTs), a majority of which were used for post-marketing safety evaluations. Further research will be conducted to determine the precise role of RWE in all studies (e.g. historical comparator, label expansion). DISCUSSION/SIGNIFICANCE OF IMPACT: By examining the use of RWE in regulatory decision making, we can inform stakeholders of the extent to which robust RWE studies complement evidence generated by RCTs. Thought to reflect a product's performance in a broader and more diverse population, RWE can provide greater insight to clinical trial conduct and ultimately transform patient outcomes.

4500

Providing a System for Practical Monitoring Training for Clinical Trials within Academic Institutions

Advaita Chandramohan ${ }^{1}$, Sukhmani Kaur ${ }^{1}$, and Eunjoo Pacifici ${ }^{1}$

${ }^{1}$ University of Southern California

OBJECTIVES/GOALS: The goal was to understand the effectiveness of a novel clinical trial educational module and a corresponding initiative designed and disseminated by the Southern California Clinical and Translational Science Institute (SC-CTSI) to increase the quality of clinical trials conducted in academia. METHODS/STUDY POPULATION: The CRCs (Clinical Research Coordinators) for the initiative are asked to complete the online training. Possible study protocols are picked to be monitored by the CRCs. The monitor is instructed to study the protocol extensively and prepare for their monitoring visit. The trained monitor from the initiative then reaches out to the CRC of the study that is to be monitored and carries out the monitoring visit. Afterwards, the monitor sends initiative personnel the monitoring report, which is evaluated to see if the monitor checked everything they should have during the visit. The PI of the study is contacted with highlights from the monitoring report and improvements that they can make. RESULTS/ ANTICIPATED RESULTS: The first study monitored was a site of a large NIH-sponsored study where the consent forms were signed electronically. It was found that the monitor could not access the consent forms. Therefore, the monitor could not do source data verification. The PI of the study said that they would be raising this issue with the NIH. During the monitoring visit of the second study chosen for the initiative, patient binders were specifically examined for informed consent and source documentation completeness. The charts of patients were also reviewed. The only deviation found was a missing signature in the Investigator Site File. For the last two studies, data will be reported. DISCUSSION/SIGNIFICANCE OF IMPACT: Monitors were not only able to monitor efficiently, but also able to point out deficiencies in the monitoring practices of large studies. This model could be expanded to other academic institutions to 
establish quality management systems to ensure data integrity and subject protection.

\section{Single IRB and the CTSI: Liaison Model for the IRB Reliance Process \\ Christine Sego Caldwell ${ }^{1}$, Amy J. Trullinger ${ }^{2}$, and Scott Denne ${ }^{1}$ ${ }^{1}$ Indiana University School of Medicine; ${ }^{2}$ Indiana CTSI, Indiana University School of Medicine}

OBJECTIVES/GOALS: Navigating the NIH Single IRB Policy has been challenging for investigators, study teams, and Human Research Protection Programs (HRPP). In response, the Indiana Clinical and Translational Sciences Institute (CTSI) created an innovative Single IRB Project Manager role (sIRB PM), uniquely placed within the Indiana CTSI. METHODS/STUDY POPULATION: The Single IRB Project Manager role was created in 2018 by the Indiana CTSI in response to the NIH Single IRB Policy for Multi-Site Research. The role of the sIRB PM is to serve as a liaison between the Indiana University HRPP, lead site, coordinating center, and participating sites when Indiana University serves as the Single IRB. This model has proven useful to both the IRB and lead site, notably in the following ways:

- At study start-up, the sIRB PM can handle complicated communications among sites and the IRB at the same time the lead site is responsible for many other administrative tasks related to start-up. By absorbing the workload of IRB approval for multiple sites, the sIRB PM provides the lead site more capacity to handle other essential tasks.

- The sIRB PM translates new terminology and facilitates processes that are new for sites.

RESULTS/ANTICIPATED RESULTS: Early assessment of this program is predominantly positive. The sIRB PM currently supports 24 external sites. In an NIA-funded 13 site study, all sites were added within 9 months of initial IRB approval of the protocol. This role fills a gap that benefits:

- IRB staff by allowing them to fulfill their duties of screening and review while leaving some of the reliance organization to the sIRBPM.

- Lead PI by allowing them to focus on conducting the research instead of the many administrative tasks required for single IRB review.

- Participating sites by having a liaison to enter their amendments and reportable events into an otherwise closed IRB software system.

- All parties by having the sIRB PM manage document organization, storage, and distribution study-wide.

DISCUSSION/SIGNIFICANCE OF IMPACT: The CTSI SIRB PM role effectively shifts administrative work caused by the sIRB mandate by merging research coordinator experience with regulatory experience while building upon an existing strong relationship with the HRPP. Future focus is on process education, standardizing pricing structure, and ensuring sufficient budget support in grants.
Survey of Regulatory Reforms to Address Comprehension of Clinical Trial Results

Matthieu Kirkland ${ }^{1}$, Christian Reyes ${ }^{1}$, Nancy Pire-Smerkanich ${ }^{1}$, and Eunjoo Pacifici ${ }^{1}$

${ }^{1}$ University of Southern California

OBJECTIVES/GOALS: Clinical research is the backbone of the medical community. However, there are few regulations to ensure clinical trial participants can understand their results, leading to volunteers feeling unvalued and unlikely to enroll in trials ${ }^{1}$. This study examines the need of lay summaries METHODS/STUDY POPULATION: To understand the current landscape of clinical trial summaries, literature searches were conducted using the University of Southern California Library database with keywords Title contains "lay language" OR "lay summary" AND any field contains "Trial" OR "clinical", and Title contains "natural language processing" AND "clinical trial" OR "Summary". Studies were deemed relevant if they discussed lay language summaries for health care realms or using Natural Language Processing (NLP) to increase comprehension. Papers published by the Center for Information and Study on Clinical Research Participation (CISCRP) were reviewed and their Associate Director was interviewed. RESULTS/ ANTICIPATED RESULTS: Of 67 total results, 14 were determined to be relevant. Ten of the relevant results examined lay language summaries and their regulation and 4 were NLP studies. The European Medicines Agency set regulations mandating clinical trial summaries. However, researchers have difficulty validating to an appropriate reading level ${ }^{2}$. Difficulty and potential bias halted a U.S. mandate of lay summaries ${ }^{3}$. The nonprofit CISCRP has partnered with industry to develop unbiased clinical trial summaries resulting in all volunteers feeling appreciated and 91\% understanding clinical trial results post summary ${ }^{1}$. Similarly, NLP software for annotating Electronic Health Records increased comprehension for $77 \%$ of patients ${ }^{4}$. DISCUSSION/SIGNIFICANCE OF IMPACT: In the U.S., a lack of regulations mandating lay summaries may be related to concerns by regulatory agencies that summaries in plain language may introduce bias $^{3}$. Future looks into integration of NLP systems to clinical trials may create unbiased summaries and allow for FDA regulation.

\section{Team Science}

4436

A Content Analysis of CTSA Websites: The Identification and Evaluation of CTSA Program Hub Website Content Standards for Knowledge Management of NCATS CTSA Program Goals and Initiatives

Barbara Ann Tafuto ${ }^{1}$, Reynold Panettieri ${ }^{1}$, James Scott Parrot ${ }^{1}$, Shankar Srinivasan ${ }^{1}$, Kristi Holmes, $\mathrm{PhD}^{2}$, and Dagobert Soergel ${ }^{3}$

${ }^{1}$ Rutgers University; ${ }^{2}$ Northwestern University; ${ }^{3}$ University of Buffalo

OBJECTIVES/GOALS: Introduction: Between 2014 and 2019 the National Institute of Health $(\mathrm{NIH})$ through the National Center for the Advancement of Translational Science (NCATS) has awarded 